\title{
ON STATE TRANSFORMATION AND STABILITY ANALYSIS OF DISTRIBUTED-PARAMETER SYSTEMS*
}

\author{
By J. A. WALKER (Northwestern University)
}

Some time ago Slemrod [1] presented an extension of LaSalle's invariance principle $[2,3,4]$ to distributed-parameter systems. As an example he considered van der Pol's nonlinear partial differential equation

$$
\ddot{u}+\epsilon\left(1-u^{2}\right) \dot{u}-u_{x x}=0, \quad t \geq 0, \quad 0 \leq x \leq 1,
$$

where $u=u(x, t), \dot{u}=\partial u / \partial t, u_{x}=\partial u / \partial x, \epsilon>0$, and the boundary conditions are $u(0, t)=u(1, t)=0, t \geq 0$.

In order to study the stability properties of a formal partial differential equation by topological (Liapunov) methods, it is necessary to replace the formal equation by an evolution equation which describes the time evolution of a quantity called the state in a metric space called the state space for all time $t \geq 0$. In [1] Eq. (1) was replaced by ${ }^{1}$

$$
\dot{u}=-v_{x}-\epsilon\left(u-u^{3} / 3\right), \quad \dot{v}=-u_{x}
$$

with the state $(u, v) \in B$ for all $t \geq 0$. A suitable state space $B$ was assumed to be $B=$ $W_{02}{ }^{1}(0,1) \times W_{2}{ }^{1}(0,1)$, where the Sobolev spaces $W_{p}{ }^{k}(\Omega)$ and $W_{02}{ }^{k}(\Omega)$ are as defined in [1], implying the natural norm

$$
\|(u, v)\|_{\beta}=\left[\int_{0}^{1}\left(u_{x}^{2}+v_{x}^{2}+u^{2}+v^{2}\right) d x\right]^{1 / 2} .
$$

The Liapunov functionals

$$
V_{\mathbb{B}}(u, v)=\int_{0}^{1}\left(u_{x}^{2}+{v_{x}}^{2}+u^{2}+v^{2}\right) d x, \quad V_{\mathbb{e}}(u, v)=\int_{0}^{1}\left(u^{2}+v^{2}\right) d x,
$$

were then sonsidered [1]. It was found that the equilibrium $(u, v)=(0,0)$ of $(2)$ is stable relative to $\|(u, v)\|_{\mathbb{Q}}$ and, in addition, $V_{\mathfrak{e}}(u, v) \rightarrow 0$ as $t \rightarrow \infty$ for all initial states $\left(u_{0}, v_{0}\right)$ such that

$$
\left\|\left(u_{0}, v_{0}\right)\right\|_{\Theta} \leq 1-\delta, \quad \delta>0 .
$$

Let us note that (2) is not the only evolution equation which may be used to represent the formal equation (1). Inasmuch as the initial data for (1) would normally be given in the form of initial values for $u$ and $\dot{u}$, it would seem more natural to replace the formal equation (1) with

$$
\dot{u}=w, \quad \dot{w}=u_{x x}-\epsilon\left(1-u^{2}\right) w
$$

* Received March 9, 1973.

${ }^{1}$ Eq. (2) is interpreted in the generalized sense [1]. 
with the state $(u, w) \in \delta$ for all $t \geq 0$. (Eq. (3) is to be interpreted in the generalized sense.) It will be shown later that a suitable state space $S$ is given by $\delta=W_{02}{ }^{1}(0,1) \times$ $W_{2}{ }^{0}(0,1)$, which has the natural norm

$$
\|(u, w)\|_{s}=\left[\int_{0}^{1}\left(u_{x}^{2}+u^{2}+w^{2}\right) d x\right]^{1 / 2} .
$$

Since (2) and (3) are representations of the same formal equation, it would seem reasonable to expect that the stability information obtained for (2) could now be carried over to (3). Unfortunately this does not appear to be the case. We note that there exists a state transformation $T, T: ß \rightarrow \mathcal{S}$, defined by ${ }^{2}$

$$
T(u, v)=\left(u,-v_{x}-\epsilon u+\epsilon u^{3} / 3\right), \quad(u, v) \in \Theta,
$$

such that every motion of (2) can be mapped into a motion of (3). (A "motion of (2)" will be defined here as a generalized solution of (2) such that $(u, v) \in \mathbb{B}$ for all $t \geq 0$. A "motion of (3)" is similarly defined.) However $T$ does not have an inverse, and therefore (3) may have a "richer" set of motions than does (2). Thus the conclusion of stability of the equilibrium of (2) can not be carried over to (3) automatically, and would not be meaningful even if it were carried over since the relevant quantity $\left\|T^{-1}(u, w)\right\|_{\Theta}$ does not exist.

The lack of an inverse for $T$ also precludes the possibility of defining new functionals

$$
\bar{V}_{a 3}(u, w)=V_{\Theta}\left(T^{-1}(u, w)\right), \bar{V}_{\mathrm{e}}(u, w)=V_{\mathrm{e}}\left(T^{-1}(u, w)\right),
$$

with a view toward direct usage of these functionals as Liapunov functionals for (3). Thus it appears that no part of the stability analysis and results for (2) can be carried over to (3).

It is significant that the difficulty we have encountered is not due to the nonlinearity of $T$, but remains even for $\epsilon=0$. Roughly speaking, the problem is that a given function $v_{x}$ on $0 \leq x \leq 1$ does not determine $v$ on $0 \leq x \leq 1$ since there is no boundary condition on $v$.

In finite-dimensional systems a state transformation $T: \varepsilon^{n} \rightarrow \mathcal{E}^{n}$ is normally assumed to be a homeomorphism, and no difficulty is encountered in transmitting stability information through such transformations. Thus one is tempted to conclude in general that state transformations must be homeomorphisms, in order for stability results to carry over. Such a restriction appears to be too strong, as we shall see by example. Noting that (3) appears to be a better representation of (1) than does (2), we shall perform a stability analysis of (3) and show that the results and analysis for (3) can be easily carried over to (2), even though the converse operation was not possible.

Consider the "energy" functional

$$
V(u, w)=\int_{0}^{1}\left(u_{x}^{2}+w^{2}\right) d x,
$$

and also the related functional [5],

$$
U(u, w)=\int_{0}^{1} u_{x}^{2} d x+\int_{0}^{1}\left[w+\epsilon\left(u-u^{3} / 3\right)\right]^{2} d x .
$$

${ }^{2} T(u, v) \in \mathcal{S}$ for $(u, v) \in B$ since $W_{2}{ }^{0}(0,1)=\mathfrak{L}_{2}(0,1)$ and, using Proposition 6.1 of $[1], u \in W_{02}{ }^{1}(0,1)$ implies $u^{k} \in \mathscr{L}_{2}(0,1)$ for any positive integer $k$. 
Assuming the existence of a sequence of smooth solutions of (1) which converge in $\delta$ uniformly in $t$, to the motion prescribed by (3) for given initial state $\left(u_{0}, w_{0}\right) \in \delta$, we compute the time derivatives of $V(u, w)$ and $U(u, w)$ according to (3) as

$$
\dot{V}(u, w)=-2 \epsilon \int_{0}^{1}\left(1-u^{2}\right) w^{2} d x, \quad \dot{U}(u, w)=-2 \epsilon \int_{0}^{1}\left(1-u^{2}\right) u_{x}^{2} d x,
$$

noting that

$$
\sup _{0 \leq x \leq 1} u^{2} \leq \int_{0}^{1} u_{x}^{2} d x
$$

Let us now consider the functional

$$
W(u, w)=V(u, w)+\alpha U(u, w),
$$

where $\alpha$ is any positive number, and note that in the subset $\mathcal{G}_{\alpha \delta}$ of $\delta$ defined by $W(u, w) \leq$ $(1+\alpha)(1-\delta), \delta>0$, we have $W(u, w)$ bounded above and below (positively) in terms of $\|(u, w)\|_{s}$, and also

$$
\dot{W}(u, w) \leq-\beta W(u, w), \quad \beta>0 .
$$

Therefore we may conclude that the equilibrium $(u, w)=(0,0)$ of $(3)$ is asymptotically stable relative to $\|(u, w)\|_{\delta}$ and the set $\zeta_{\alpha \delta}$ defines a domain of attraction for any $\alpha>0$, $\delta>0$. It is also apparent that $\delta$ is an appropriate state space for (3) when the initial state lies in $\oint_{\alpha \delta}$, since any motion originating in $\oint_{\alpha \delta}$ must remain in $\oint_{\alpha \delta}$ (and hence in $\delta$ ) for all $t>0[6, \mathrm{p} .52]$.

We will now show that the analysis and results for (3) can be carried over to (2) via the transformation $T$ of (4). Consider the functionals defined on $\&$ by

$$
\begin{aligned}
& \bar{V}(u, v)=V(T(u, v))=\int_{0}^{1} u_{x}^{2} d x+\int_{0}^{1}\left[v_{x}+\epsilon\left(u-u^{3} / 3\right)\right]^{2} d x, \\
& \bar{U}(u, v)=U(T(u, v))=\int_{0}^{1}\left(u_{x}^{2}+v_{x}^{2}\right) d x .
\end{aligned}
$$

The derivatives of these functionals according to (2) can be obtained either by direct calculation according to (2), or by noting that every motion of (2) defines a motion of (3) via the transformation $T$ and that therefore we must have

$$
\begin{aligned}
& \dot{\bar{V}}(u, v)=\dot{V}(T(u, v))=-2 \epsilon \int_{0}^{1}(1-u)^{2}\left[v_{x}+\epsilon\left(u-u^{3} / 3\right)\right]^{2} d x, \\
& \dot{\bar{U}}(u, v)=\dot{U}(T(u, v))=-2 \epsilon \int_{0}^{1}\left(1-u^{2}\right) u_{x}^{2} d x .
\end{aligned}
$$

We now consider the functional defined by

$$
\begin{aligned}
\bar{W}(u, v) & =W(T(u, v)) \\
& =\bar{V}(u, v)+\alpha \bar{U}(u, v), \quad \alpha>0,
\end{aligned}
$$

and note that in the subset $\overline{\mathcal{G}}_{\alpha \delta}$ of $\beta$ defined by

$$
\bar{W}(u, v) \leq(1+\alpha)(1-\delta), \quad \delta>0,
$$


we have $\bar{W}(u, v)$ bounded above and below (positively) in terms of $\|(u, v)\|_{\otimes}$, and also

$$
\dot{\bar{W}}(u, v) \leq-\beta \bar{W}(u, v), \quad \beta>0 .
$$

Therefore we may conclude that the equilibirum $(u, v)=(0,0)$ of $(2)$ is asymptotically stable relative to $\|(u, v)\|_{\beta}$ and the set $\overline{\mathcal{S}}_{\alpha \delta}$ defines a domain of attraction for any $\alpha>0$, $\delta>0$. We note that $T \overline{\mathcal{G}}_{\alpha \delta}=\mathcal{G}_{\alpha \delta}$ and that within $\overline{\mathcal{G}}_{\alpha \delta}$ the quantity $\|T(u, v)\|_{s}$ is bounded above and below (positively) in terms of $\|(u, v)\|_{a}$.

Thus we have been able to carry the stability results and analysis for (3) over to (2) in a one-to-one manner, even though the transformation $T$ is not one-to-one. We were successful because $T$ embeds the dynamical system $[1,6,7]$ described by $(2)$ on $\overline{\mathcal{S}}_{\alpha \delta}$ into the dynamical system described by (3) on $\oint_{\alpha \delta}$, and the property we wished to carry over from (3) to (2) was asymptotic stability. Had the equilibrium of (3) proved to be unstable but not completely unstable, it might not have been possible to transfer such a conclusion to (2).

It appears that state transformation (and definition) must be performed with considerable care if one hopes to perform a stability analysis of one system which will have meaning for the other system. It also appears that this point may have important implications for the mathematical modeling of physical distributed-parameter systems [8].

\section{REFERENCES}

[1] M. Slemrod, Asymptotic behavior of a class of abstract dynamical systems, J. Diff. Eqs. 7, 584-600 (1970)

[2] J. P. LaSalle, Asymptotic stability criteria, in Proceedings of symposia in applied mathematics 13, AMS, Providence, R. I., 1962.

[3] J. P. LaSalle, An invariance principle in the theory of stability, in Differential equations and dynamical systems, ed. J. K. Hale and J. P. LaSalle, Academic Press, 1967.

[4] J. P. LaSalle, Stability theory for ordinary differential equations, J. Diff. Eqs. 4, 57-65 (1968)

[5] J. A. Walker, Energy-like Liapunov functiorials for linear elastic systems on a Hilbert space, Quart. Appl. Math. 30, 46:-480 (197:3)

[6] J. K. Hale, Dynamical systems and stability, J. Math. Anal. Appl. 26, 39-59 (1969)

[7] V. I. Zubov, Methods of A. M. Liapunov and their applications, Noordhoff, 1964

[8] J. A. Walker, Lagrange's equation for continua, J. Math. Anal. Appl. (to appear) 